\title{
Professional mechanical plaque removal alone may not be enough to maintain gingival health
}

\author{
Abstracted from \\ Needleman I, Nibali L, Di lorio A. \\ Professional mechanical plaque removal for prevention of periodontal diseases in adults \\ - systematic review update. / Clin Periodontol 2015; 42: S12-35. doi: 10.1111/jcpe.12341. PubMed PMID: 25495962. \\ Address for correspondence: Ian Needleman, Unit of Periodontology, Eastman Dental Institute, \\ University College London, (UCL) University of London, 256 Gray's Inn Road, \\ London, WC1X 8LD, UK. E-mail: i.needleman@ucl.ac.uk
}

\section{Question: What is the effect of professional mechanical plaque removal on clinical and patient reported outcomes related to the primary prevention of periodontal diseases in adults?}

Data sources The Cochrane Central Register of Controlled Trials (CENTRAL), Medline and Embase databases.

Study selection Randomised controlled trials of professional plaque removal with a comparison group of no intervention, oral hygiene instruction only or different frequencies of professional mechanical plaque removal were considered.

Data extraction and synthesis Independent duplicate data abstraction was carried out by two reviewers. Included studies were assessed with the Cochrane risk of bias tool. Meta-analysis was not conducted because of marked heterogeneity in the included studies. Results Three studies were included. Risk of bias was unclear or high in all three studies. Three studies compared professional mechanical plaque removal (PMPR) and oral Hygiene Instruction $(\mathrm{OHI})$ versus no treatment and found evidence for PMPR and $\mathrm{OHI}$ achieving more favourable changes in plaque and bleeding/inflammation compared with no treatment. There was no available evidence for an effect on pocket depth (PD) or attachment level (AL). Two studies compared $\mathrm{PMPR}+\mathrm{OHI}$ versus $\mathrm{OHI}$ and found evidence suggesting no difference between $\mathrm{PMPR}+\mathrm{OHI}$ and $\mathrm{OHI}$ alone in maintaining or improving plaque and bleeding outcomes. There was no additional information from other intervention comparisons in the three studies. Evidence from the three new studies was considered with existing RCT evidence. Conclusions Within the limitations of the research, we suggest the following conclusions: low-moderate strength of evidence suggests that in adults, PMPR, particularly if combined with $\mathrm{OHI}$, may achieve greater changes in measures of dental plaque and gingival bleeding/ inflammation than no treatment. Moderate strength of evidence suggests there is no additional benefit to plaque and gingival bleeding outcomes from PMPR over that achieved by repeated and thorough oral hygiene instructions, although with no evidence to inform on prevention of periodontitis. Low strength of evidence suggests that more frequent PMPR is associated with improved plaque and bleeding outcomes and possibly less annual attachment loss.

\section{Commentary}

Since the 1700s mechanical removal of plaque and calculus (ie scaling and/or polishing) together with personal plaque control has been the standard of care to prevent gingivitis, and ultimately periodontitis. In most developed countries today, apart from a dental examination, professional mechanical plaque removal (PMPR) is the most common service delivered by dental clinicians. ${ }^{1}$ However, the scientific evidence supporting the use of PMPR to prevent periodontitis has been weak or non-existent. ${ }^{2}$

Needleman's rigourous systematic review is an update of the 2005 review, ${ }^{3}$ and follows Cochrane protocol. It examines the evidence for PMRP with and without oral hygiene instruction on prevention of periodontal diseases. Despite the ten years since the last review, only three new studies met the inclusion criteria. The evidence suggests that while PMPR may influence gingival health, repeated, thorough OHI may have as great an impact. Thus, professional plaque removal without good daily personal plaque control is likely of limited value. The overall strength of evidence was moderate (meaning further research may change the estimate of effect one way or another), with consistency of findings across studies.

As pointed out in the review, the applicability of the evidence to clinical practice must be met with some scepticism. Firstly, there are no data investigating the prevention of periodontitis itself. Multi-year, multi-site studies with large enough sample sizes are in short supply. These studies are also necessary to investigate the potential interaction between PMPR and OHI. Further, a lack of sufficient evidence of efficacy (does something work in a controlled setting like an RCT?) does not necessarily mean it does not work in everyday clinical practice. For those determinations, long-term observational studies examining appropriate outcomes (tooth loss, patient-based outcomes) may be helpful.

The aetiology of periodontal diseases is multifactorial, and not everyone is at risk of progressive attachment loss. However, personal plaque control is often seen as one of the key modifiable risk factors. Traditionally, dental professionals have applied the biomedical model of disease prevention - disseminating health education and providing expert advice. There is strong evidence from a high quality systematic review that this is ineffective. ${ }^{4}$ This may be because this model ignores the fact that oral health behaviours are inextricably linked to the behaviours people use to cope with other personal, social and economic influences in their lives. There is good evidence to support motivational interviewing (MI) as an effective model for chair-side oral health promotion. Nevertheless, this technique takes time (which is not always appropriately 
reimbursed by dental health insurance) and training (which is not necessarily included in all curricula, particularly for dentists). The advantage of MI is that it allows practitioners a way to tailor $\mathrm{OHI}$ to be adapted to the individual patient's needs and degree of readiness to change behaviours.

One of the key messages in the recent Scottish Dental Clinical Effectiveness Programme (SDCEP) guidance document ${ }^{5}$ on periodontal health is that management of periodontal health is a partnership between patient and clinician and requires a life-long commitment. Certainly evidence from Needleman's review supports the fact that engaging patients in decision-making around their oral health care may be as important to maintenance of periodontal health as many of the mechanical and pharmacologic therapies we use.
Debora Matthews

Faculty of Dentistry, Dalhousie University Halifax,

Nova Scotia, Canada

1. Brown LJ, Johns BA, Wall TP. The economics of periodontal diseases. Periodontol 2000 2002; 29: 223-234.

2. Worthington HV, Clarkson JE, Bryan G, Beirne PV. Routine scale and polish for periodontal health in adults. Cochrane Database Syst Rev 2013; 11: CD004625.

3. Needleman I, Suvan J, Moles DR, Pimlott J. A systematic review of professional mechanical plaque removal for prevention of periodontal diseases. / Clin Periodontol 2005; 32: 229-282.

4. Yevlahova D, Satur J. Models for individual oral health promotion and their effectiveness: a systematic review. Aust Dent / 2009; 54: 190-197.

5. Prevention and Treatment of Periodontal Diseases in Primary Care, Dental guidance. Scottish Dental Clinical Effectiveness Programme, Dundee, June 2014.

Evidence-Based Dentistry (2015) 16, 74-75. doi:10.1038/sj.ebd.6401112 